\title{
Simulación en laparoscopía avanzada con un modelo de anastomosis entero-entérica
}

\author{
Dres. Javier Chinelli, Gustavo Rodríguez ${ }^{\dagger}$
}

\section{Resumen}

Introducción: la enseñanza de la cirugía atraviesa un cambio de paradigma, siendo el entrenamiento en laparoscopía avanzada uno de sus mayores desafíos. El objetivo de este estudio es describir y evaluar la experiencia de un servicio de cirugía universitario con un modelo de entrenamiento simulado de anastomosis intestinal laparoscópica.

Método: tres residentes de cirugía general completaron un programa de entrenamiento de cuatro semanas. Se utilizó un modelo biológico ex vivo en box trainer, evaluando objetivamente la realización de una anastomosis entero-entérica.

Resultados: el tiempo de ejecución del procedimiento se redujo en una media de 15 minutos, con una mejoría significativa del desempeño según la escala OSATS.

Discusión: la implementación de un programa validado y modificado de simulación en laparoscopía avanzada permitió obtener resultados positivos, utilizando para ello solo el $4 \%$ de la carga horaria semanal curricular. El modelo tiene una alta fidelidad, bajo costo y es fácilmente reproducible.

Conclusiones: el entrenamiento simulado en laparoscopía es una herramienta obligatoria y beneficiosa durante la formación del cirujano general.

Palabras clave: Laparoscopía

Simulación

Anastomosis quirúrgica

Intestinos

Anastomosis intestinal

Key words: Laparoscopía

Simulation

Anastomosis, surgical

Intestines

Bowel anastomosis

\footnotetext{
* Asistente, Clínica Quirúrgica 2.

† Profesor Titular, Clínica Quirúrgica 2.

Clínica Quirúrgica 2. Hospital Maciel.

Correspondencia: Dr. Javier Chinelli. Correo electrónico: Jchinelli01@gmail.com

No existen conflictos de interés ni apoyo de la industria.

Aprobado por el Comtié de Ética del Hospital Maciel

Recibido: 25/9/19

Aprobado: $17 / 2 / 20$
} 


\section{Introducción}

La enseñanza de la cirugía se basó tradicionalmente en el modelo de Halsted ${ }^{(1)}$, que supuso un gran avance en la formación de los residentes. En el modelo se combinaban las clases magistrales, junto a la enseñanza a los pies de la cama del paciente, y también en la sala de operaciones. Los alumnos avanzaban observando los procedimientos en primer lugar, asistiendo como ayudantes en segunda instancia y finalmente realizándolos ("see one, do one, teach one"), asignándoles entonces una responsabilidad progresiva.

Actualmente se asiste a un cambio de paradigma en relación con el modelo de enseñanza motivado por varios factores, que podemos sintetizar de la siguiente manera: avances en teoría educativa ${ }^{(2,3)}$; factores éticos relacionados, entre otras cuestiones, con la seguridad del paciente $^{(4)}$, y desafíos propios de la cirugía miniinvasiva como la visión bidimensional, pérdida de sensación táctil y curvas de aprendizaje más largas en general.

La simulación, ampliamente utilizada desde principios del siglo XX en el campo de la aviación, encuentra su lugar dentro de la enseñanza moderna de habilidades técnicas en cirugía. Su principal ventaja, entre otras, es la de crear un entorno seguro y controlado ${ }^{(5)}$.

Recientemente hemos revisado los principales aspectos relacionados a los diferentes modelos de simulación utilizados habitualmente en cirugía (inertes, vivos, cadavéricos, de realidad virtual), así como nuestra experiencia inicial de entrenamiento de habilidades básicas con modelos de banca y material inanimado ${ }^{(6)}$.

El principal objetivo de esta investigación es evaluar la aplicación de una metodología previamente validada de entrenamiento simulado en habilidades laparoscópicas avanzadas en un servicio de cirugía universitario, utilizando un modelo de anastomosis entero-entérica laparoscópica.

Como objetivo secundario, determinar si el entrenamiento descrito permite alcanzar resultados positivos en la adquisición de habilidades técnicas.

\section{Material y método}

Estudio prospectivo, observacional y analítico. Participaron tres residentes de cirugía general de segundo año, que previamente habían completado un entrenamiento en habilidades quirúrgicas básicas sobre modelos inertes en box trainer. Las actividades que a continuación se describen se llevaron a cabo en el laboratorio de cirugía del Hospital Maciel entre agosto y setiembre de 2019.

Simulador: se utiliza un modelo de banca o box trainer. Se trata de un dispositivo que simula un abdomen, con orificios para la introducción de trócares e instru- mental laparoscópico. Posee una cámara con transmisión a un monitor situado frente al alumno.

Modelo de entrenamiento: el modelo es biológico e inanimado (tejidos ex vivo). Consiste en intestino delgado bovino previamente acondicionado mediante lavado y conservación a baja temperatura, con lo que permanece viable durante algunos días.

Programa de entrenamiento: el programa consiste en la realización de una anastomosis entero-entérica laparoscópica simulada orientada verticalmente (figura 1).

Antes de iniciar el entrenamiento, un cirujano del equipo del servicio de cirugía (Clínica Quirúrgica 2) demostró detalladamente el procedimiento a los participantes, instruyéndolos en aspectos fundamentales: instrumental a utilizar, materiales de sutura y la técnica a seguir. Se utilizan portaagujas, pinza Maryland, tijera e hilo de sutura poliglactina 910 (Vicryl) 3-0. Inicialmente se solicitó a los participantes que realizaran una anastomosis tal como les fuera demostrado, cuya evaluación se detalla más adelante.

La práctica simulada de la anastomosis tiene como base la deconstrucción de ésta en cuatro pasos y la práctica deliberada o repetitiva de cada uno de éstos, que son los siguientes: tres puntos de aproximación sero-musculares, dos enterotomías longitudinales, sutura de pared posterior y sutura de pared anterior.

Se llevaron a cabo dos sesiones de entrenamiento semanales de 60 minutos de duración cada una y se estableció una práctica secuencial, de modo tal que a medida que los alumnos progresan, se van agregando pasos sucesivos de la técnica para culminar realizando la anastomosis completa. El período de entrenamiento fue de cuatro semanas, procediendo luego a la evaluación final (tabla 1).

Se proporcionó un feedback efectivo a cargo de un instructor (cirujano de staff), de tipo formativo a lo largo del entrenamiento, y de tipo sumativo al final.

Evaluación del desempeño: tanto durante la realización de la primera anastomosis como en la última (evaluación final) se consideraron los siguientes aspectos:

- Tiempo de ejecución (minutos).

- Longitud adecuada de la anastomosis: $5 \mathrm{~cm}$.

- Distancia adecuada entre 2 puntos: 2,5 - 4,5 mm. Para ello, se toma como referencia el punto medio de la línea de sutura en la cara anterior y la distancia al punto siguiente.

- Eversión o no de la mucosa

- Aplicación de la escala Objective Structured Assessment of Technical Skills (OSATS) ${ }^{(7)}$ (figura 2). 


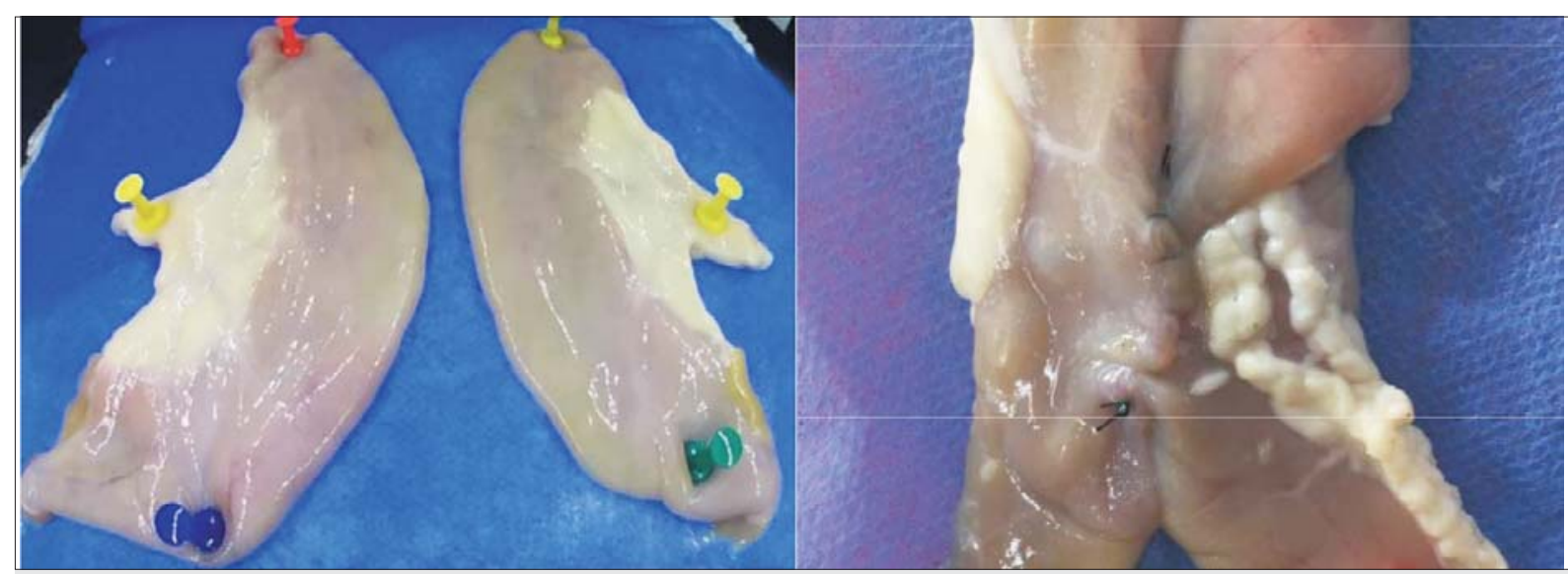

Figura 1. Modelo de entrenamiento y anastomosis completa. Fuente: autor

\begin{tabular}{|c|c|c|c|c|c|}
\hline Puntuación & Respeto por los tejidos & Tiempo y movimientos & Uso del instrumento & $\begin{array}{l}\text { Tiempo de cirugia y } \\
\text { planificación posterior }\end{array}$ & Conocimientos \\
\hline 1 & $\begin{array}{l}\text { Uso frecuente de fuerza } \\
\text { innecesaria. Daño } \\
\text { causado por uso } \\
\text { inapropiado de los } \\
\text { instrumentos }\end{array}$ & $\begin{array}{l}\text { Muchos movimientos } \\
\text { innecesarios }\end{array}$ & $\begin{array}{l}\text { Repetidamente hace } \\
\text { movimientos vacilantes y } \\
\text { torpes con instrumentos }\end{array}$ & $\begin{array}{l}\text { Frecuentemente detiene } \\
\text { el procedimiento } 0 \\
\text { requiere discutir los } \\
\text { pasos siguientes }\end{array}$ & $\begin{array}{l}\text { Conocimiento deficiente y } \\
\text { requiere instrucciones en } \\
\text { la mayoria de los pasos } \\
\text { quirürgicos }\end{array}$ \\
\hline 3 & $\begin{array}{l}\text { Manejo cuidadoso de los } \\
\text { tejidos, pero } \\
\text { ocasionalmente produce } \\
\text { daño inadvertido }\end{array}$ & $\begin{array}{l}\text { Relación tiempo y } \\
\text { movimientos eficiente, } \\
\text { pero realiza algunos } \\
\text { movimientos } \\
\text { innecesarios }\end{array}$ & $\begin{array}{l}\text { Uso competente de los } \\
\text { instrumentos, pero } \\
\text { ocasionalmente se } \\
\text { observa rígido o torpe }\end{array}$ & $\begin{array}{l}\text { Demuestra habilidades } \\
\text { para planear los pasos } \\
\text { siguientes, con } \\
\text { progresión constante del } \\
\text { procedimiento }\end{array}$ & $\begin{array}{l}\text { Conoce todos los } \\
\text { aspectos importantes de } \\
\text { la operación }\end{array}$ \\
\hline 5 & $\begin{array}{l}\text { Maneja los tejidos } \\
\text { apropiadamente con } \\
\text { minimo daño }\end{array}$ & $\begin{array}{l}\text { Economia de } \\
\text { movimientos y máxima } \\
\text { eficiencia }\end{array}$ & $\begin{array}{l}\text { Movimientos fluidos con } \\
\text { los instrumentos y sin } \\
\text { torpeza }\end{array}$ & $\begin{array}{l}\text { Curso planeado de la } \\
\text { operación en forma } \\
\text { obvia, con flujo sin } \\
\text { esfuerzo }\end{array}$ & $\begin{array}{l}\text { Familiaridad demostrada } \\
\text { con todos los aspectos } \\
\text { de la operación }\end{array}$ \\
\hline
\end{tabular}

Figura 2. Escala global OSATS. Tomado de Martin JA, et al. Br J Surg 1997; 84:273-8.

\section{Resultados}

Los tres participantes realizaron el programa de entrenamiento siguiendo el cronograma estipulado a lo largo de cuatro semanas consecutivas, con una carga individual total de 8 horas.

La anastomosis inicial demandó un tiempo promedio de 43,3 min (40-45), con una media de 6,3 puntos en la escala OSATS (6-7). En los tres casos la longitud de la misma fue correcta, señalándose como principal error técnico una distancia inadecuada entre los puntos (mayor a $4,5 \mathrm{~mm}$ en los tres casos).

Luego del entrenamiento, se evaluó la anastomosis final. El tiempo promedio empleado fue de 28 minutos (27-30 min), con una media de 16,3 puntos en la escala OSATS (16-17). En relación con los detalles técnicos, todos los participantes obtuvieron una distancia media adecuada entre puntos de $3,2 \mathrm{~mm}(3-3,5 \mathrm{~mm})$ y $\sin$ eversión mucosa.
Los principales resultados se presentan en la tabla 2.

\section{Discusión}

La implementación de la simulación en laparoscopía en el campo de la cirugía general es algo relativamente novedoso en Uruguay. Al momento de comunicar nuestra experiencia inicial en el entrenamiento de habilidades laparoscópicas básicas en residentes de primer y segundo año de cirugía general ${ }^{(6)}$, no existían referencias bibliográficas de autores nacionales en relación con este tema. Por otra parte, la formación del cirujano en la actualidad nos enfrenta a importantes desafíos, como la necesidad de continuar enseñando procedimientos por vía abierta, al tiempo que la cirugía laparoscópica gana cada vez más terreno acorde a los constantes avances tecnológicos. Si a esto le sumamos que para la última existen curvas de aprendizaje más largas en comparación con el abordaje convencional, el problema adquie- 
Tabla 1. Distribución de las sesiones de entrenamiento.

\begin{tabular}{|c|c|c|}
\hline Semana & Día 1 & Día 2 \\
\hline 1 & $S A \times 3$ & $S A \times 3$ \\
\hline \multirow[t]{3}{*}{2} & $S A \times 2$ & $S A \times 2$ \\
\hline & Ent $\times 2$ & Ent $\times 2$ \\
\hline & & $P P \times 2$ \\
\hline \multirow[t]{4}{*}{3} & $S A \times 2$ & $\mathrm{SA} \times 1$ \\
\hline & Ent $\times 2$ & Ent $x 1$ \\
\hline & PP x2 & $\mathrm{PP} \times 1$ \\
\hline & & PA $\times 1$ \\
\hline 4 & $\begin{array}{l}\text { Anastomosis } \\
\text { completa (1) }\end{array}$ & $\begin{array}{l}\text { Anastomosis } \\
\text { completa (final) }\end{array}$ \\
\hline
\end{tabular}

SA: suturas de aproximación (3); Ent: enterotomías; PP: pared posterior; PA: pared anterior

re una dimensión particularmente importante en nuestro medio, en el que por cuestiones demográficas los centros hospitalarios (incluso los universitarios) son de bajo volumen. Es justamente por ello que surge nuestro interés en esta temática, la simulación, entendida como la técnica de imitar una conducta, situación, procedimiento o proceso, por medio de un aparato o escenario adecuado, con propósitos de estudio o entrenamiento ${ }^{(8)}$.

En este estudio participaron tres residentes de segundo año del posgrado de especialista en cirugía general que previamente habían completado un programa validado de simulación en adquisición de habilidades laparoscópicas básicas fundado en ejercicios de los programas Yale Laparoscopic Skills and Suturing Program ${ }^{(9)}$ y Fundamentals of Laparoscopic Surgery ${ }^{(10)}$. De esta forma, se asegura un nivel mínimo de destrezas necesario para el programa propuesto, a la vez que se trata de alumnos sin experiencia previa en la ejecución de procedimientos de laparoscopía avanzada, por lo que consideramos que se encuentran en una etapa formativa especialmente adecuada para los objetivos descritos.

De forma global, podemos decir que los resultados de esta investigación han sido positivos.

En primer lugar, queda demostrado que es perfectamente posible implementar un programa de entrenamiento simulado en laparoscopía avanzada en un servicio de cirugía universitario si se dispone de los recursos e infraestructura necesarios. El primero de ellos, una planta física adecuada. Actualmente, el Hospital Maciel cuenta con un laboratorio de cirugía en el que hemos desarrollado ésta y otras experiencias similares en mode-
Tabla 2. Tiempo de ejecución de las anastomosis y evaluación objetiva.

\begin{tabular}{|ccccc}
\hline & $\begin{array}{c}\text { Tiempo } \\
\text { anastomosis } \\
\text { inicial (min) }\end{array}$ & $\begin{array}{c}\text { Tiempo } \\
\text { anastomosis } \\
\text { final (min) }\end{array}$ & OSATS inicial & OSATS final \\
\hline R1 & 45 & 27 & 7 & 16 \\
R2 & 40 & 27 & 7 & 17 \\
R3 & 45 & 30 & 6 & 16 \\
\hline
\end{tabular}

los de tipo box trainer. Si tomamos en cuenta el tiempo semanal destinado al programa (dos horas), éste supone solo el $4 \%$ del total de horas por semana que establece la currícula del residente de cirugía general en la actualidad (48 horas) ${ }^{(11)}$, con la ventaja adicional de poder realizarlo en el propio hospital, sin necesidad de tener que trasladarse a otro centro. El intestino bovino ex vivo utilizado para entrenar las anastomosis intestinales, si bien no permite su reutilización, presenta como principales ventajas una alta fidelidad y bajo costo, además de bioseguridad.

En segundo lugar, porque efectivamente se aprecia un avance en la adquisición de habilidades laparoscópicas avanzadas, tal como lo demuestra una reducción de 15 min en el tiempo medio de ejecución de la anastomosis (cuya realización en manos de un cirujano entrenado no debería ser mayor a 20 min en total), así como en la calidad de la misma, con un incremento promedio de 10 puntos en la escala OSATS. Se ha demostrado la relación entre la puntuación obtenida con esta escala y el desempeño posterior realizando la misma anastomosis en la sala de operaciones ${ }^{(12)}$. Esto es el resultado de un enfoque de tipo constructivista en la concepción del entrenamiento. Para ello, primero debe deconstruirse el procedimiento a realizar (anastomosis intestinal en este caso) en sus pasos fundamentales, metodología que se conoce como entrenamiento parcial de tareas o partial task trai$n_{i n g}{ }^{(13)}$. Se comienza entrenando el primer paso repetidamente y una vez que éste ha sido dominado y puede ejecutarse con proficiencia, se agrega un segundo paso al anterior (que se sigue ejecutando), y así sucesivamente hasta dominar por completo el procedimiento. De esta forma, se agregan tareas nuevas solo cuando la anterior se lleva a cabo de forma completamente automatizada

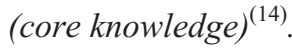

Esta metodología, conocida como práctica deliberada (deliberate practice), propuesta por Ericsson ${ }^{(15)}$, hace referencia a una modalidad de entrenamiento que se caracteriza por la práctica repetitiva con uno o más objetivos concretos, que en general suelen estar algo por encima de las posibilidades reales de ser alcanzados. Re- 
quiere de alumnos motivados y en conocimiento de las áreas en que deben mejorar, la asignación de objetivos y tareas concretas, un feedback inmediato, y un entorno simulado $^{(16)}$.

Varas y colaboradores presentaron los resultados de un ensayo ${ }^{(17)}$ en el que la aplicación de este programa de entrenamiento simulado permitió demostrar no solamente la posibilidad de ser aplicado en cualquier laboratorio de simulación, sino además la mejoría significativa en la adquisición de habilidades (a un nivel equivalente al de experto) por parte de un grupo de residentes de diversas especialidades quirúrgicas. Por si ello fuera poco, también demostraron la transferencia de dichas habilidades a un segundo escenario simulado (modelo animal vivo), lo que permitió establecer la validez del método empleado. Nuestra experiencia toma como base dicha metodología de simulación (simuladores, modelo biológico, deconstrucción, entrenamiento secuencial y constructivista, evaluación inicial y final utilizando escalas, y parámetros objetivos), pero destinando una carga horaria sensiblemente reducida con respecto a ella. Esto responde a limitantes y dificultades propias del medio en el que pretendemos desarrollarla: disponibilidad semanal limitada del centro de simulación y la coordinación con otras actividades docentes y asistenciales del staff del servicio. Aun así, consideramos que los resultados obtenidos han sido aceptables, destacando que el tiempo de ejecución de un procedimiento considerado de laparoscopía avanzada se redujo, en promedio, 38\% luego del entrenamiento.

Este trabajo tiene algunas debilidades que deben analizarse para interpretar con cautela sus principales resultados y que se relacionan entre sí. El número de participantes es bajo, y si bien todos ellos fueron instruidos previamente de la misma forma en cuanto a habilidades básicas, hay una variabilidad individual relacionada a la capacidad de adquirir destrezas (básicas y avanzadas). Esta variabilidad determina que las curvas de aprendizaje sean distintas para cada caso, y no necesariamente todos ellos puedan alcanzar los objetivos planteados con el cronograma establecido, máxime si tenemos en cuenta que éste toma la base de un programa validado, pero al que hemos reducido la carga horaria y la distribución de las sesiones prácticas. En segundo lugar, la evaluación de la técnica, aunque hecha en base a parámetros objetivos bien definidos, no fue ciega y carece de la medición de una variable como es la presión de fuga de la anastomosis, la que se determina conectando la misma a un manómetro y proporciona un parámetro objetivo más confiable que el de la distancia entre puntos para predecir el riesgo de filtración.

\section{Conclusiones}

De acuerdo a nuestra experiencia, es factible aplicar la simulación en un modelo de entrenamiento avanzado y los resultados obtenidos son alentadores.

\section{Summary}

Background: there is a changing paradigm in surgical education, being laparoscopic training one of its major challenges. The objective of this study is to describe and evaluate our experience with a simulated laparoscopic small bowel anastomosis training model at a universitary surgical center.

Methods: a 4-week training program was conducted with participation of 3 general surgery residents. An ex vivo biological model in a box trainer was used to objectively evaluate a simulated entero-enterostomy.

Results: final procedure time was reduced an average of 15 minutes, with better outcomes according to OSATS scale.

Discussion: implementation of a modified, validated advanced laparoscopic skills training program showed positive results, taking only $4 \%$ of the weekly curricular schedule. The model has high fidelity, low cost and is easily reproducible.

Conclusions: simulated laparoscopic training is both mandatory and beneficial in surgical education.

\section{Resumo}

Introdução: o ensino da cirurgia atravessa um cambio de paradigma, sendo o treinamento em laparoscopia avançada um de sus maiores desafios. O objetivo deste estudo é descrever e avaliar a experiencia de um serviço universitário de cirurgia com um modelo de treinamento simulado de anastomose intestinal laparoscópica.

Métodos: 3 residentes de cirurgia general completaram um programa de treinamento de 4 semanas. Foi empregado um modelo biológico ex vivo em simuladores de caixa, avaliando objetivamente a realização de uma anastomose entero-entérica.

Resultados: o tempo de execução do procedimento foi reduzido em média 15 minutos, com una melhoria significativa do desempenho segundo a escala OSATS.

Discussão: a implementação de um programa validado e modificado de simulação em laparoscopia avançada permitiu obter resultados positivos, utilizando somente $4 \%$ da carga horaria semanal curricular. O modelo tem alta fidelidade, baixo custo e é facilmente reproduzível.

Conclusões: o treinamento simulado em laparoscopia é uma ferramenta obrigatória e benéfica durante a formação do cirurgião geral. 


\section{Bibliografía}

1. Carter BN. The fruition of Halsted's concept of surgical training. Surgery 1952; 32:518-27.

2. Fitts PM, Posner MI. Human performance. Belmont, CA: Brooks/Cole, 1967.

3. Kolb DA. Experiential learning: experience as the source of learning and development. Englewood Cliffs, NJ: Prentice Hall, 1984.

4. Kohn LT, Corrigan JM, Donaldson MS, eds. To err is human: building a safer health system. Washingtpn, DC: National Academies Press, 2000.

5. Reznick R, MacRae H. Teaching surgical skills-changes in the wind. N Engl J Med 2006; 355:2664-9.

6. Chinelli J, Rodriguez G. Simulación en laparoscopía durante la formación del cirujano general. Revisión y experiencia inicial. Rev Méd Urug 2018; 34(4):232-9.

7. Martin JA, Regehr G, Reznick R, MacRae H, Murnaghan J, Hutchison C, et al. Objective structured assessment of technical skill (OSATS) for surgical residents. Br J Surg 1997; 84:273-8.

8. Bradley $\mathbf{P}$. The history of simulation in medical education and possible future directions. Med Educ 2006; 40(3):254-62.

9. Scott DJ, Bergen PC, Rege RV, Laycock R, Tesfay ST, Valentine RJ, et al. Laparoscopic training on bench models: better and more cost effective than operating room experience? J Am Coll Surg 2000; 191(3):272-83.

10. Soper NJ, Fried GM. The fundamentals of laparoscopic surgery: its time has come. Bull Am Coll Surg 2008; 93(9):30-2.

11. Universidad de la República, Facultad de Medicina, Escuela de Graduados. Programa de formación de especia- listas en cirugía general. Montevideo: Facultad de Medicina-UdelaR, 2016. 15p. Disponible en: http://www.fmed. edu.uy/sites/www.egradu.fmed.edu.uy/files/Programa_de_postgrados/Especialidades/CIRUG\%C3\%8DA\%20GENERAL.pdf [Consulta: 14 diciembre 2019].

12. de Montbrun SL, Roberts PL, Lowry AC, Ault GT, Burnstein MJ, Cataldo PA, et al. A novel approach to assessing technical competence of colorectal surgery residents: the development and evaluation of the Colorectal Objective Structured Assessment of Technical Skill (COSATS). Ann Surg 2013; 258(6):1001-6.

13. Kolozsvari NO, Feldman LS, Vassiliou MC, Demyttenaere S, Hoover ML. Sim one, do one, teach one: considerations in designing training curricula for surgical simulation. J Surg Edu 2011; 68(5):421-7.

14. Hirsch ED, Jr. The core knowledge curriculum-what's behind its success? Educ Leadership 1993; 50(8):23-30.

15. Ericsson KA, Krampe RT, Tesch-Römer C. The role of deliberate practice in the acquisition of expert performance. Psychol Rev 1993; 100(3):363-406.

16. McGaghie WC, Issenberg SB, Barsuk JH, Wayne DB. A critical review of simulation-based mastery learning with translational outcomes. Med Educ 2014; 48(4):375-85.

17. Varas J, Mejía R, Riquelme A, Maluenda F, Buckel E, Salinas $\mathbf{J}$, et al. Significant transfer of surgical skills obtained with an advanced laparoscopic training program to a laparoscopic jejunojejunostomy in a live porcine model: feasibility of learning advanced laparoscopy in a general surgery residency. Surg Endosc 2012; 26:3486-94.

\section{Contribución de autores}

Ambos autores participaron en igual medida en todas las etapas de elaboración del artículo

Javier Chinelli 0000-0002-3387-7365

Gustavo Rodríguez 0000-0003-3465-8364 